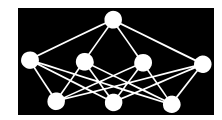

\title{
FLOPPY LOGIC - A YOUNGER SISTER OF FUZZY LOGIC
}

\author{
P. Provinsky*
}

\begin{abstract}
This article introduces a floppy logic - a new method of work with fuzzy sets. This theory is a nice connection between the logic, the probability theory and the fuzzy sets. The floppy logic has several advantages compared to the fuzzy logic: All propositions, which are equivalent in the bivalent logic, are equivalent in the floppy logic too. Logical operations are modeled unambiguously, not by using many alternative t-norms and t-conorms. In floppy logic, we can use the whole apparatus of Kolmogorov's probability theory. This theory allows to work consistently with systems that are described by fuzzy sets, probability distributions and accurate values simultaneously.
\end{abstract}

Key words: floppy logic, floppy sets, fuzzy logic, fuzzy sets, probability, Kolmogorov's axioms, floppy probability space

Received: October 6, 2015

DOI: $10.14311 / \mathrm{NNW} .2017 .27 .025$

Revised and accepted: October 30, 2017

\section{Introduction}

This article introduces a "floppy logic" - a new method of work with fuzzy sets.

In this Introduction we provide basic information to facilitate orientation in this text.

In the second section we will discuss very briefly the fuzzy logic and probability theory. There are the following facts interesting for us: Fuzzy sets are very useful and successful tools for a system description. The fuzzy logic is not compatible with the probability theory. There are many t-norms and t-conorms which can be alternatively a generalization of sets union and intersection.

In the third section we present the basic floppy logic. We will introduce basic floppy sets, their probabilities and membership functions. That section finishes with many examples.

The fourth section is a generalization of the basic floppy logic. That generalization allows us to use fuzzy statements and precise statements together. A nice feature of the floppy logic is explained in that part: All statements, which are equivalent in standard bivalent logic, are equivalent in floppy logic too. There are many examples at the end of the section too.

\footnotetext{
*Pavel Provinský; Czech Technical University in Prague, Faculty of Transportation Sciences, Na Florenci 25, Praha 110 00, Czech Republic, E-mail: provipav@fd.cvut.cz
} 
Then the conclusion and two appendices follow: overview of the Kolmogorov's axioms and proofs of the submitted theorems.

The basic idea of the floppy logic is this: If a system of primary fuzzy sets fulfills appropriate assumptions, then it meets all Kolmogorov's axioms and it is, therefore, a model of Kolmogorov's probability theory. Then we can work with fuzzy sets as with objects of probability theory. ${ }^{1}$

\section{Fuzzy logic and probability theory}

\subsection{Probability theory}

The emergence of the probability theory falls to the mid 17th century and is connected with the names of Blaise Pascal, Pierre de Fermat and Christian Huygens. A nice treatise on its history can be found in [18] or [24]. An important step was the axiomatization of the theory carried out by Andrey Nikolajevic Kolmogorov in 1933 in the book [15]. This axiomatization led to a great development of the theory and related disciplines. Another generalization of the axioms was performed by Alfred Renyi in [23]. A big advantage of probability theory is an unambiguous mathematical apparatus.

\subsection{History of fuzzy sets}

We can read about a history of fuzzy sets in the book [8]. The notion "fuzzy set" first appeared in 1965 in Lotfi Zadeh's article [28]. A fundamental idea of the sets whose elements could have a degree of membership in the whole range from zero to one is presented in that paper. Subsequently, the author solves the second question how to generalize the standard intersection and union for these sets. Lotfi Zadeh already notes in this work, that there are several operations that can be such a generalization. Firstly, he presents the maximum and minimum of membership functions, secondly the algebraic sum and algebraic product. Zadeh ascribes the discovery of the algebraic sum as a dual operations to the algebraic product to $\mathrm{T}$. Cover. An operation which we call "algebraic sum", is called the "sum" by Zadeh simply. The name "algebraic sum" he used for the simple sum of membership functions.

The third pair of such operations is the bounded sum and the bounded difference, which appeared in Robin Giles' work [12] from 1976. Some other operations followed soon. Generally, we can generalize the intersection for crisp sets by any triangular norm and the union by any triangular conorms.

The term "triangular norm" first appeared in 1942 in Karl Menger's work [19]. Within the context of fuzzy sets, this term is firstly used in Didier Dubois and Henri M. Prade's book [9] including the important proposition

$$
T_{w}(a, b) \leq T(a, b) \leq \min (a, b),
$$

where $T(a, b)$ is any triangular norm and $T_{w}(a, b)$ is the drastic product.

\footnotetext{
${ }^{1}$ I took over this idea from Ivan Nagy, the supervisor of my dissertation.
} 
The set operations union and intersection correspond to the logical operations disjunctions and conjunctions. So we can have many operations, triangular norms and conorms, which generalize conjunction and disjunction of classical logic. If we want to realize e.g. a fuzzy controller, we would face the task to choose one from many logical operations. This ambiguity is a disadvantage of the fuzzy set theory.

On the other hand, a fuzzy description is similar to natural human language and systems can be described by fuzzy rules easily and comfortably.

\subsection{The conjunction of probability theory and fuzzy sets}

The idea to connect the probability theory and the fuzzy set theory is not new. The probability and also the degree of membership have values within the same range $\langle 0,1\rangle$. This immediately suggests the question whether the two theories are not equivalent. Almost every popular text about fuzzy sets emphasizes that they are not the same. For example, Wikipedia in the entry "Fuzzy logic" states: "Fuzzy logic and probability address different forms of uncertainty." See [10].

Both approaches, however, can be combined in many ways. E.g. in article [30], Lotfi Zadeh introduces a classical probability of fuzzy phenomena. ${ }^{2}$ In [27], the same author already thinks about the probability of fuzzy events, which is not given by a real number but by a fuzzy number. The conditional probability of fuzzy objects is the subject of paper [21].

\subsection{The differences between the probability theory and the theory of fuzzy sets}

The basic differences between the probability theory and the theory of fuzzy sets which are often referred, are two:

1) Both of these theories describe different types of uncertainty.

We can meet the interpretation of membership functions as a degree of similarity (e.g. [2]), a degree of preference (e.g. [3]) or a degree of uncertainty (e.g. [4]). The membership function can be often seen as a conditional probability $P(A \mid x)$ where a given element $x$ will be assigned to the set $A$ by a queried subject (e.g. by an expert). This approach can be found e.g. in [14].

Note that although these theories describe different types of uncertainty, it does not mean automatically that these uncertainty cannot be described by the same axiomatic theory.

2) The membership function of an intersection or union of two fuzzy sets depends only on the membership functions of these sets. We say, that fuzzy logic is "functional".

On the other hand, the probability of the intersection or union of two events depends not only on the probabilities of these events, but also on their conditional probability. The probability theory is not "functional".

\footnotetext{
${ }^{2}$ However, he does not show under what conditions this way defined probability satisfies the Kolmogorov's axioms.
} 


\subsection{The relations between the probability theory and the theory of fuzzy sets}

An interesting relationship can be found in the article [11] where Brian R. Gaines introduces the "uncertainty logic". It leads, after adding the law of excluded middle, to Rescher's probabilistic logic which is introduced in the book [22]. On the contrary, addition of the requirement of functionality of logic leads to Lukasiewicz's fuzzy logic. The addition of both requirements together leads to contradiction.

Another relationship between probability theory and fuzzy sets can be found in similar properties of conditional probability on one hand and fuzzy relative cardinality on the other. This similarity was pointed out by Bart Kosko in the article [17].

Here we must mention the possibility theory, developed by Lotfi Zadeh [29], Didier Dubois and Henri M. Prade [7], which lies between the probability theory and theory of fuzzy sets. Events have assigned two numbers in this theory: possibility and necessity.

The possibility and the necessity have two properties similar to membership functions of fuzzy sets.

1) The possibility of union of any two events is equal to the maximum of the possibility of these events.

2) The necessity of intersection of any two events is equal to the minimum of the necessity of these events.

On the other hand, there is also a link to the probability theory. The possibility, namely, can be understood as an upper probability envelope.

I would like to mention one recent result about the relationship between the probability theory and the theory of fuzzy sets. The authors of the paper [20] study a question, which t-norms and t-conorms in conjunction with Zadeh's definition of probability of fuzzy events [30] meet Kolmogorov's axioms.

Some other ideas about fuzzy logic and probability can be found in [13] or [1].

Many references dealing with the relationship between the probability theory and the fuzzy sets can be found in the compendious article [6].

\section{Basic floppy logic}

\subsection{Basic floppy sets}

Which assumptions must be fulfilled in order to obtain a probability space?

Let $\boldsymbol{S}$ be a system of primary fuzzy sets $A_{i}$.

A set of all subsets of $\boldsymbol{S}$ will be denoted by $\mathcal{P}(\boldsymbol{S})$.

The definition domain of the membership functions of fuzzy sets from $\boldsymbol{S}$ will be denoted by $X$.

The membership functions of fuzzy sets $A_{i}$ will be denoted by $\mu_{A_{i}}(x)$, where $x \in X$. We can often write shortly $\mu_{A_{i}}$.

We will assume that the definition domain $X$ and the system of fuzzy sets $\boldsymbol{S}$ satisfy these assumptions:

Assumption 1. $\boldsymbol{S}$ is a finite or countable set. 
Assumption 2. Membership functions of fuzzy sets $A_{i} \in \boldsymbol{S}$ assume values from the interval $\langle 0,1\rangle$.

Assumption 3. The sum of all the membership functions of fuzzy sets $A_{i} \in \boldsymbol{S}$ is equal to one everywhere:

$$
\forall x \in X: \sum_{A_{i} \in S} \mu_{A_{i}}(x)=1 .
$$

Assumption 4. A measure space $(X, \mathcal{A}, P)$ is defined on the set $X$ where $\mathcal{A}$ is a $\sigma$-algebra on $X$ and $P$ is a probability measure.

Assumption 5. All membership functions $\mu_{A_{i}}$ of fuzzy sets $A_{i} \in \boldsymbol{S}$ are measurable on the sets $X_{i} \in \mathcal{A}$ with respect to the measure $P$.

Now we can introduce next definitions:

Definition 1. Sets of primary fuzzy sets will be called basic floppy sets and will be denoted by bold capital letters.

E.g. subsets of $\boldsymbol{S}$ are basic floppy sets.

Definition 2. The membership function of basic floppy set $\boldsymbol{B} \subseteq \boldsymbol{S}$ is defined by the rule:

$$
\boldsymbol{\mu}_{\boldsymbol{B}}(x)=\sum_{A_{i} \in \boldsymbol{B}} \mu_{A_{i}}(x)
$$

where $x \in X$.

The membership function of basic floppy sets will be denoted by the bold letter $\mu$.

The membership function $\boldsymbol{\mu}_{\boldsymbol{B}}(x)$ is defined well. See Lemmas 3 and 4 , Subsection 7.1.

Note, that we have membership functions of fuzzy sets and membership functions of basic floppy sets and it is very important to distinguish between them.

Definition 3. A probability $Q$ of basic floppy set $\boldsymbol{B} \subseteq \boldsymbol{S}$ is defined by the rule:

$$
Q(B)=\int_{X} \boldsymbol{\mu}_{\boldsymbol{B}}(x) d P
$$

where the integral on the right side is the Lebesgue integral with a probability measure $P$ over the set $X$.

This definition is similar but not equivalent to Zadeh's definition of probability of fuzzy events from [30]. A question, which t-norm and t-conorms connected with Zadeh's definition of probability of fuzzy events satisfy Kolmogorov's axioms, is very complicated [20]. On the other hand, the same question with floppy sets and our new definition of probability is very simple.

The probability $Q(\boldsymbol{B})$ is defined well. See Lemma 6, Subsection 7.1.

If we wanted to work only with discrete random variables, we could define the number $Q(\boldsymbol{B})$ as follows:

$$
Q(B)=\sum_{x_{i} \in X} \boldsymbol{\mu}_{\boldsymbol{B}}\left(x_{i}\right) \cdot P\left(x_{i}\right) .
$$


If we wanted to work only with continuous random variables with probability density function $f(x)$, we could define the number $Q(\boldsymbol{B})$ using the Riemann integral as follows:

$$
Q(B)=\int_{X} \boldsymbol{\mu}_{\boldsymbol{B}}(x) \cdot f(x) d x .
$$

Our definition using Lebesgue integral covers both of these cases, and combinations thereof.

Definition 4. The space $(\boldsymbol{S}, \mathcal{P}(\boldsymbol{S}), Q)$ will be called a basic floppy probability space.

The names "probability of basic floppy set" in the Definition 3 and "basic floppy probability space" in the Definition 4 are justified through the next theorem:

Theorem 1. About basic floppy probability spaces

Each basic floppy probability space satisfies all Kolmogorov's axioms.

The proof of Theorem 1 is placed in Subsection 7.2.

Thus, the basic floppy sets can be seen as probability events, the set $\boldsymbol{S}$ as a sample space, and so on.

\section{$3.2 \quad$ Examples}

Is this system a basic floppy probability space?

We describe a temperature of water by three fuzzy sets: hot $(H)$, tepid $(T)$ and cold $(C)$ with membership functions $\mu_{H}, \mu_{T}$ a $\mu_{C}$. The temperature will be denoted by letter $x$ and the probability density function of temperature occurrence by symbol $f(x)$. The membership functions and the density function are figured in Figure 1. ${ }^{3}$

Does this system satisfy Assumptions 1 to 5 ?

1. $\boldsymbol{S}=\{H, T, C\}$ is a finite set.

2. Values of membership functions are from the interval $\langle 0,1\rangle$.

3. The sum of membership function is 1 everywhere on $X$.

4. There is some probability density function of temperature occurrence, so there is defined some probability space $(X, \mathcal{A}, P)$.

5. All membership functions are simple and measurable on the set $X$ with respect to the measure $P$.

Therefore $(\boldsymbol{S}, \mathcal{P}(\boldsymbol{S}), Q)$ is a basic floppy probability space.

Now we are looking for the membership functions and the probability of floppy events:

\footnotetext{
${ }^{3}$ The left vertical axis is valid for membership functions, the right for the probability density function.
} 
1. A singleton of fuzzy set - The water is hot.

$$
\begin{aligned}
\boldsymbol{\mu}_{\{H\}} & =\mu_{H}, \\
Q(\{H\}) & =\int_{X} \boldsymbol{\mu}_{\{H\}} d P=\int_{0}^{100} \mu_{H}(x) \cdot f(x) d x .
\end{aligned}
$$

2. A negation - The water is not hot.

$$
\begin{aligned}
\boldsymbol{\mu}_{\overline{\{H\}}} & =\boldsymbol{\mu}_{\{T, C\}}=\mu_{T}+\mu_{C} \\
Q(\overline{\{H\}}) & =\int_{X} \boldsymbol{\mu}_{\overline{\{H\}}} d P=\int_{0}^{100}\left(\mu_{T}+\mu_{C}\right) \cdot f(x) d x
\end{aligned}
$$

3. A conjunction - The water is not hot and is not cold.

$$
\begin{aligned}
\boldsymbol{\mu}_{\overline{\{H\}} \cap \overline{\{C\}}}=\boldsymbol{\mu}_{\{T, C\} \cap\{H, T\}}=\boldsymbol{\mu}_{\{T\}}=\mu_{T}, \\
Q(\overline{\{H\}} \cap \overline{\{C\}})=\int_{X} \boldsymbol{\mu}_{\overline{\{H\}} \cap \overline{\{C\}}} d P=\int_{0}^{100} \mu_{T} \cdot f(x) d x .
\end{aligned}
$$

4. A disjunction - The water is hot or tepid.

$$
\begin{aligned}
\boldsymbol{\mu}_{\{H\} \cup\{T\}} & =\boldsymbol{\mu}_{\{H, T\}}=\mu_{H}+\mu_{T}, \\
Q(\{H\} \cup\{T\}) & =\int_{X} \boldsymbol{\mu}_{\{H\} \cup\{T\}} d P=\int_{0}^{100}\left(\mu_{H}+\mu_{T}\right) \cdot f(x) d x .
\end{aligned}
$$

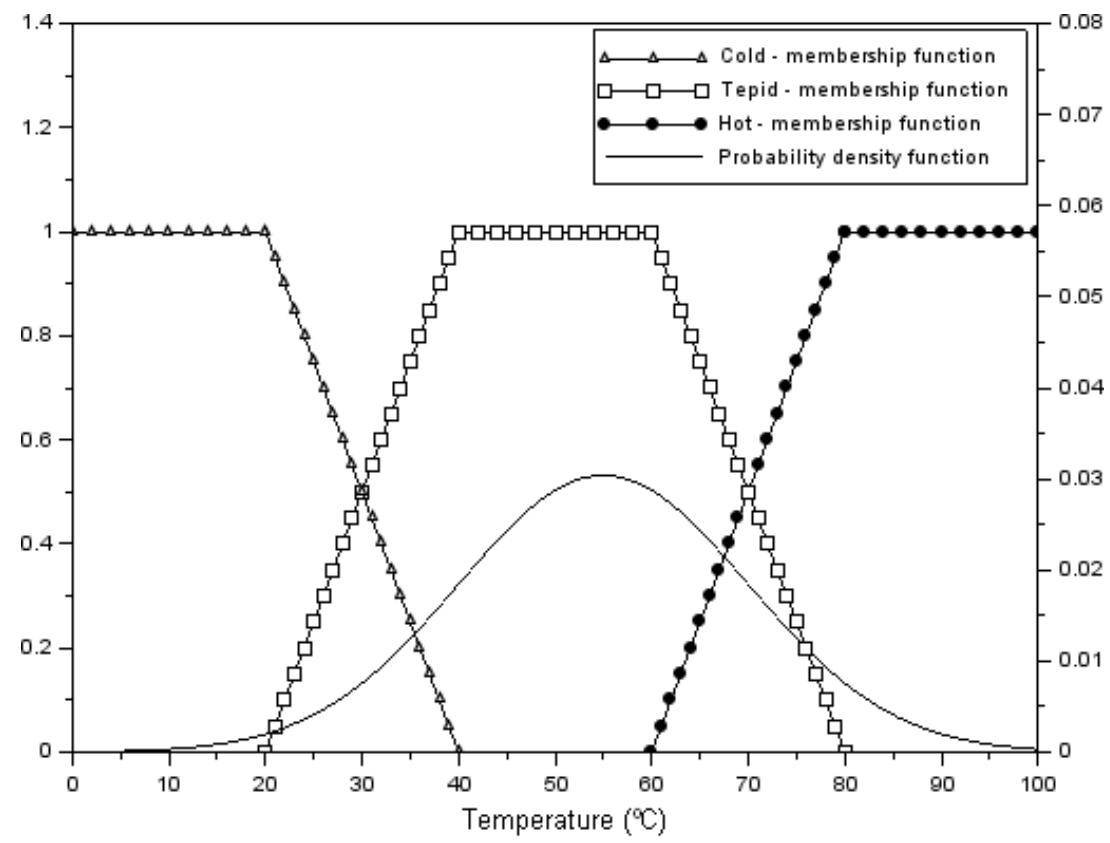

Fig. 1 The membership functions and the probability density function. 
5. An implication - If the water is hot, then it is not cold.

An equivalence $H \Rightarrow \neg C \longleftrightarrow \neg H \vee \neg C$ is used. The equivalence of floppy statements will be discussed in Subsection 4.2.

$$
\begin{aligned}
\boldsymbol{\mu}_{\overline{\{H\}} \cup \overline{\{C\}}} & =\boldsymbol{\mu}_{\{T, C\} \cup\{H, T\}}= \\
& =\boldsymbol{\mu}_{\{H, T, C\}}=\mu_{H}+\mu_{T}+\mu_{C}=1 \\
Q(\overline{\{H\}} \cup \overline{\{C\}}) & =\int_{X} \boldsymbol{\mu}_{\overline{\{H\}} \cup \overline{\{C\}}} d P=\int_{0}^{100} 1 \cdot f(x) d x=1 .
\end{aligned}
$$

6. An equivalence - The water is hot if and only if it is cold.

An equivalence $H \Leftrightarrow C \longleftrightarrow(H \wedge C) \vee(\neg H \wedge \neg C)$ is used.

$$
\begin{aligned}
\boldsymbol{\mu}_{(\{H\} \cap\{C\}) \cup(\overline{\{H\}} \cap \overline{\{C\}})} & =\boldsymbol{\mu}_{\varnothing \cup\{T\}}= \\
& =\boldsymbol{\mu}_{\{T\}}=\mu_{T}, \\
Q((\{H\} \cap\{C\}) \cup(\overline{\{H\}} \cap \overline{\{C\}})) & =\int_{X} \boldsymbol{\mu}_{\{T\}} d P= \\
& =\int_{0}^{100} \mu_{T} \cdot f(x) d x=Q(\{T\}) .
\end{aligned}
$$

In the floppy logic, we can use every relations from Kolmogorov's probability theory. For example:

7. The formula for the union of events - What is the probability that the water is tepid or is not hot?

$$
\begin{aligned}
Q(\{T\} \cup \overline{\{H\}})= & Q(\{T\})+Q(\overline{\{H\}})-Q(\{T\} \cap \overline{\{H\}})= \\
= & \int_{X} \mu_{T} \cdot f(x) d x+\int_{X}\left(\mu_{T}+\mu_{C}\right) \cdot f(x) d x- \\
& -\int_{X} \mu_{T} \cdot f(x) d x= \\
= & \int_{0}^{100}\left(\mu_{T}+\mu_{C}\right) \cdot f(x) d x .
\end{aligned}
$$

8. The definition of the conditional probability - What is the probability that the water is hot, provided that is not cold?

$$
\begin{aligned}
Q(\{H\} \mid \overline{\{C\}}) & =\frac{Q(\{H\} \cap \overline{\{C\}})}{Q(\overline{\{C\}})}= \\
& =\frac{Q(\{H\})}{Q(\{H, T\})}=\frac{\int_{0}^{100} \mu_{H} \cdot f(x) d x}{\int_{0}^{100}\left(\mu_{H}+\mu_{T}\right) \cdot f(x) d x} .
\end{aligned}
$$


9. The Bayes' theorem - What is the probability that the water is not cold, provided that is hot?

$$
\begin{aligned}
Q(\overline{\{C\}} \mid\{H\}) & =\frac{Q(\{H\} \mid \overline{\{C\}}) \cdot Q(\overline{\{C\}})}{Q(\{H\})}= \\
& =\frac{\frac{\int_{X} \mu_{H} \cdot f(x) d x}{\int_{X}\left(\mu_{H}+\mu_{T}\right) \cdot f(x) d x} \cdot \int_{X}\left(\mu_{H}+\mu_{T}\right) \cdot f(x) d x}{\int_{X} \mu_{H} \cdot f(x) d x}=1 .
\end{aligned}
$$

\section{Generalized floppy logic}

\subsection{Floppy sets}

We have two probabilistic spaces now. The space $(X, \mathcal{A}, P)$ was assumed and the space $(\boldsymbol{S}, \mathcal{P}(\boldsymbol{S}), Q)$ was defined. Let us create a joint probability space.

We need a sample space, $\sigma$-algebra rich enough, and probabilistic measure. We assume fulfillment of Assumptions 1 to 5, Subsection 3.1.

The sample space of that joint probability space will be the Cartesian product $\boldsymbol{S} \times X$.

Definition 5. The smallest $\sigma$-algebra over $\mathcal{P}(\boldsymbol{S}) \times \mathcal{A}$ will be denoted $\mathcal{C}$.

Definition 6. Elements of $\mathcal{C}$ will be called floppy sets.

The floppy sets will be denoted by bold capital letters.

Definition 7. A membership function of floppy set $\boldsymbol{C} \in \mathcal{C}$ is defined by the rule:

$$
\boldsymbol{\mu}_{\boldsymbol{C}}(x)=\sum_{A_{i} \in \boldsymbol{S}:\left[A_{i}, x\right] \in \boldsymbol{C}} \mu_{A_{i}}(x) .
$$

The membership function of floppy sets will be denoted by bold letter $\boldsymbol{\mu}$.

Definition 8. A probability $R$ of floppy set $\boldsymbol{C} \in \mathcal{C}$ is defined by the rule:

$$
R(\boldsymbol{C})=\int_{X} \boldsymbol{\mu}_{\boldsymbol{C}}(x) d P
$$

Definition 9. The space $(\boldsymbol{S} \times X, \mathcal{C}, R)$ will be called a floppy probability space.

The names "probability of floppy set" in the Definition 8 and "floppy probability space" in the Definition 9 are justified through the next theorem:

Theorem 2. About floppy probability spaces

Each floppy probability space satisfies all Kolmogorov's axioms.

The proof of Theorem 2 is placed in Subsection 7.3.

Thus, floppy sets can be seen as probabilistic events and it is possible to work with them this way. 


\subsection{Equivalent statements in floppy logic}

Statements equivalent in the standard logic are equivalent in the floppy logic too.

In what sense are the floppy statements equivalent?

Statements in the standard logic can be modeled by crisp sets. Two equivalent statements correspond to the same set.

Statement in the floppy logic are represented by floppy sets i.e. by crisp sets. Thus two equivalent statement correspond to the same floppy set and their membership functions are the same.

\subsection{Examples}

For simplicity, sometimes $\boldsymbol{B}$ will be written instead of $\boldsymbol{B} \times X, x$ instead of $\boldsymbol{S} \times x$, and so on. Sometimes logical operators will be written instead of sets operators.

1. A simple floppy set - The water is hot and the temperature is under $90{ }^{\circ} \mathrm{C}$.

$$
\begin{gathered}
\boldsymbol{\mu}_{\boldsymbol{C}}(x)=\sum_{A_{i} \in \boldsymbol{S}:\left[A_{i}, x\right] \in \boldsymbol{C}} \mu_{A_{i}}(x)= \begin{cases}\mu_{H} & x \in\langle 0,90\rangle, \\
0 & x \in(90,100\rangle .\end{cases} \\
R(\boldsymbol{C})=\int_{0}^{90} \mu_{H}(x) d P=\int_{0}^{90} \mu_{H}(x) \cdot f(x) d x .
\end{gathered}
$$

2. A bit complicated floppy set - The water is cold or tepid and the temperature is over $30^{\circ} \mathrm{C}$ or the water is hot or tepid and the temperature is under $80^{\circ} \mathrm{C}$.

$$
\begin{gathered}
\boldsymbol{\mu}_{\boldsymbol{C}}(x)=\sum_{A_{i} \in \boldsymbol{S}:\left[A_{i}, x\right] \in \boldsymbol{C}} \mu_{A_{i}}(x)= \begin{cases}\mu_{H}+\mu_{T} & x \in\langle 0,30), \\
\mu_{C}+\mu_{T}+\mu_{H}=1 & x \in\langle 30,80\rangle, \\
\mu_{C}+\mu_{T} & x \in(80,100\rangle .\end{cases} \\
\begin{aligned}
R(\boldsymbol{C})=\int_{0}^{30}\left(\mu_{H}(x)+\mu_{T}(x)\right) d P+\int_{30}^{80} d P+ \\
=\int_{20}^{30} \mu_{T}(x) \cdot f(x) d x+\int_{30}^{80} f(x) d x+0 .
\end{aligned}
\end{gathered}
$$

3. The equivalence between sets $\boldsymbol{B} \subseteq \boldsymbol{S}$ and $\boldsymbol{B} \times X \in \mathcal{C}-$ The water is hot.

$$
\boldsymbol{\mu}_{\boldsymbol{B} \times X}(x)=\mu_{H}(x)=\boldsymbol{\mu}_{\boldsymbol{B}}(x),
$$

where $x \in\langle 0,100)$.

$$
\begin{aligned}
R(\boldsymbol{B} \times X) & =\int_{0}^{100} \mu_{H}(x) d P= \\
& =\int_{0}^{100} \mu_{H}(x) \cdot f(x) d x=Q(\boldsymbol{B})
\end{aligned}
$$


4. A crisp set - The temperature of water is from the interval $\langle 50,90\rangle^{\circ} \mathrm{C}$.

$$
\begin{gathered}
\boldsymbol{\mu}_{\boldsymbol{C}}(x)=\sum_{A_{i} \in S:\left[A_{i}, x\right] \in \boldsymbol{C}} \mu_{A_{i}}(x)= \begin{cases}0 & x \in\langle 0,50), \\
\mu_{C}+\mu_{T}+\mu_{H}=1 & x \in\langle 50,90\rangle, \\
0 & x \in(90,100\rangle .\end{cases} \\
R(\boldsymbol{C})=\int_{50}^{90} d P=\int_{50}^{90} f(x) d x .
\end{gathered}
$$

5. An implication - If the temperature is from interval $\langle 80,100\rangle^{\circ} \mathrm{C}$, then the water is hot. $=$ The water is hot or the temperature is not from the interval $\langle 80,100\rangle^{\circ} \mathrm{C}$.

$$
\begin{aligned}
\boldsymbol{\mu}_{\boldsymbol{C}}(x) & =\sum_{A_{i} \in \boldsymbol{S}:\left[A_{i}, x\right] \in \boldsymbol{C}} \mu_{A_{i}}(x)= \begin{cases}1 & x \in\langle 0,80), \\
\mu_{H} & x \in\langle 80,100\rangle .\end{cases} \\
R(\boldsymbol{C}) & =\int_{0}^{80} 1 d P+\int_{80}^{100} \mu_{H}(x) d P= \\
& =\int_{0}^{80} f(x) d x+\int_{80}^{100} \mu_{H}(x) \cdot f(x) d x=1 .
\end{aligned}
$$

6. A conditional probability. We use the standard definition of the conditional probability from the probability theory. - What is the probability that the water is hot, provided that the temperature is from the interval $\langle 80,100\rangle^{\circ} \mathrm{C}$ ?

$$
\begin{aligned}
R(\{H\} \mid\langle 80,100\rangle) & =\frac{R(\{H\} \cap\langle 80,100\rangle)}{R(\langle 80,100\rangle)}= \\
& =\frac{\int_{80}^{100} \mu_{H}(x) d P}{\int_{80}^{100} d P}= \\
& =\frac{\int_{80}^{100} \mu_{H}(x) \cdot f(x) d x}{\int_{80}^{100} f(x) d x}=1 .
\end{aligned}
$$

7. A relation between an implication $\boldsymbol{C} \Rightarrow \boldsymbol{D}$ and a conditional probability $R(\boldsymbol{D} \mid \boldsymbol{C})$.

$$
R(\boldsymbol{C} \Rightarrow \boldsymbol{D})=R(\overline{\boldsymbol{C}})+R(\boldsymbol{C} \cap \boldsymbol{D})=1-R(\boldsymbol{C})+R(\boldsymbol{D} \mid \boldsymbol{C}) \cdot R(\boldsymbol{C}) .
$$

So, if $R(\boldsymbol{D} \mid \boldsymbol{C})=1$, then $R(\boldsymbol{C} \Rightarrow \boldsymbol{D})=1$.

If $R(\boldsymbol{C} \Rightarrow \boldsymbol{D})=1$ and $R(\boldsymbol{C}) \neq 0$, then $R(\boldsymbol{D} \mid \boldsymbol{C})=1$. 


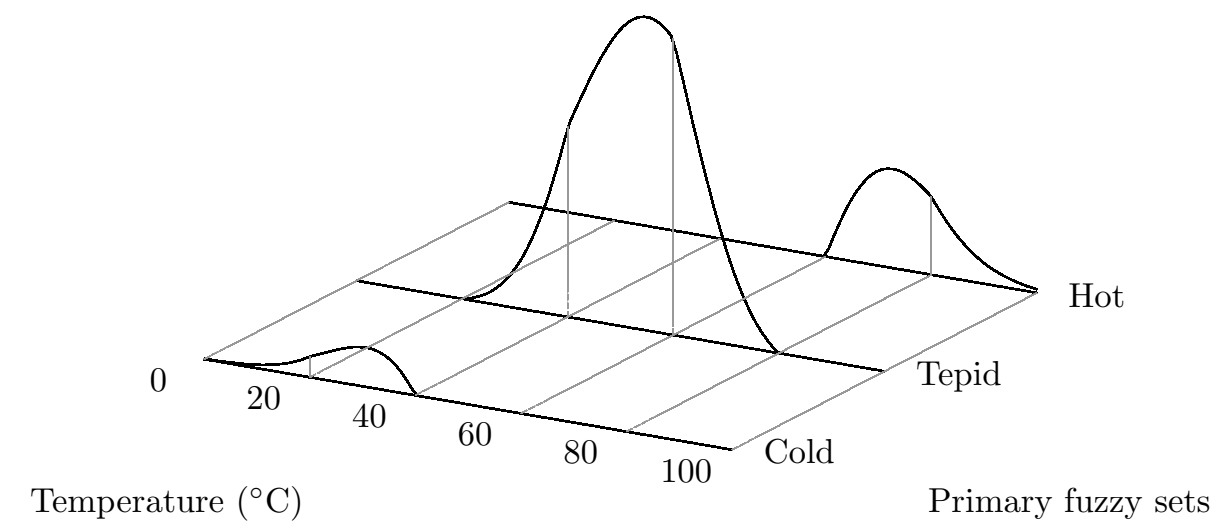

Fig. 2 The joint probability density function

8. The joint probability density function - What is the probability density function at the point $\left[A_{i}, x\right]$ ?

The joint probability density function is given by the equation:

$$
f\left(A_{i}, x\right)=\mu_{A_{i}}(x) \cdot f(x),
$$

where $A_{i} \in \boldsymbol{S}$.

See Fig. 2 where the joint probability density function is shown.

9. A sense of the conditional probability $R(\boldsymbol{C} \mid x)$ where $\boldsymbol{C} \in \mathcal{C}$ and $x \in X$. We use the conditional probability definition from the probability theory. What is the probability that the water is hot, provided that the temperature is $x$ ?

$$
\begin{aligned}
R(\boldsymbol{C} \mid x) & =\frac{f(\boldsymbol{C} \cap x)}{f(x)}= \\
& =\frac{\boldsymbol{\mu}_{\boldsymbol{C}}(x) \cdot f(x)}{f(x)}= \\
& =\boldsymbol{\mu}_{\boldsymbol{C}}(x)=\mu_{H}(x) .
\end{aligned}
$$

10. The Bayes' theorem - What is the probability density function of tempera- 
ture, provided that the water is hot?

$$
\begin{aligned}
f(x \mid \boldsymbol{C}) & =\frac{R(\boldsymbol{C} \mid x) \cdot f(x)}{R(\boldsymbol{C})}=\frac{\boldsymbol{\mu}_{\boldsymbol{C}}(x) \cdot f(x)}{\int_{X} \boldsymbol{\mu}_{\boldsymbol{C}}(x) \cdot f(x) d x}= \\
& =\frac{\mu_{H}(x) \cdot f(x)}{\int_{0}^{100} \mu_{H}(x) \cdot f(x) d x} .
\end{aligned}
$$

11. A mean value - What is the temperature mean value of hot water?

$$
\begin{aligned}
E(x \mid \boldsymbol{C}) & =\int_{X} x \cdot f(x \mid \boldsymbol{C}) d x= \\
& =\frac{1}{R(\boldsymbol{C})} \cdot \int_{X} x \cdot \boldsymbol{\mu}_{\boldsymbol{C}}(x) \cdot f(x) d x= \\
& =\frac{1}{R(\{H\})} \cdot \int_{0}^{100} x \cdot \mu_{H}(x) \cdot f(x) d x .
\end{aligned}
$$

Thus, each floppy set can be represented by the number $E(x \mid \boldsymbol{C})$. This number will be denoted $\langle\boldsymbol{C}\rangle$ and called the mean value of floppy set $\boldsymbol{C}$.

12. What is the mean value of numbers $\left\langle\left\{A_{i}\right\}\right\rangle$ ?

$$
\begin{aligned}
E\left(\left\langle\left\{A_{i}\right\}\right\rangle\right) & =\sum_{\forall i}\left\langle\left\{A_{i}\right\}\right\rangle \cdot R\left(\left\{A_{i}\right\}\right)= \\
& =\sum_{\forall i} \int_{X} x \cdot \boldsymbol{\mu}_{\left\{A_{i}\right\}}(x) \cdot f(x) d x= \\
& =\int_{X} x \cdot f(x) d x=E(x) .
\end{aligned}
$$

13. Contraposition of implication - If the water is hot, then the temperature is over $60^{\circ} \mathrm{C}$. $\Longleftrightarrow$ If the temperature is not over $60^{\circ} \mathrm{C}$, then the water is not hot.

$$
\boldsymbol{\mu}_{\{H\} \Rightarrow\langle 60,100\rangle}=\boldsymbol{\mu}_{\overline{\{H\}} \cup\langle 60,100\rangle}=\boldsymbol{\mu}_{\langle 60,100\rangle \cup \overline{\{H\}}}=\boldsymbol{\mu}_{\neg\langle 60,100\rangle \Rightarrow \neg\{H\}} .
$$

14. De Morgan's law - It is not true that the water is hot or colder than $20^{\circ} \mathrm{C}$. $\Longleftrightarrow$ It is not true that the water is hot and it is not true that the water is colder than $20^{\circ} \mathrm{C}$.

$$
\boldsymbol{\mu}_{\overline{\{H\} \cup\langle 0,20\rangle}}=\boldsymbol{\mu}_{\overline{\{H\}} \cap \overline{\langle 0,20\rangle}}= \begin{cases}0 & x \in\langle 0,20), \\ \mu_{T}+\mu_{C} & x \in\langle 20,100\rangle .\end{cases}
$$

\section{Conclusion}

In this article, we introduced the theoretical foundations of the floppy logic and showed some examples.

We have proved that system of primary fuzzy sets, which fulfills the appropriate conditions, is a model of Kolmogorov's probability theory.

Therefore, the floppy sets can be understood as probabilistic events and it is possible to work with them this way. 


\section{Appendix - Kolmogorov's axioms}

We take over Kolmogorov's axioms from the books [15, 25] in this form:

We have a set of values, which can be taken by a random variable. This set will be denoted by $\Omega$ and called sample space.

Next we have the $\sigma$-algebra $\mathcal{B}$ of subsets of $\Omega$. Thus we assume:

Axiom 1. $\varnothing \in \mathcal{B}$.

Axiom 2. If $A \in \mathcal{B}$, then $\bar{A} \in \mathcal{B} .^{4}$

Axiom 3. If $A_{1}, A_{2}, \ldots, A_{n}, \ldots \in \mathcal{B}$, then

$$
\bigcup_{i=1}^{\infty} A_{i} \in \mathcal{B}
$$

The elements of $\mathcal{B}$ will be called events.

A real number $P(A)$ will be assigned to all events $A \in \mathcal{B}$. The number $P(A)$ will be called the probability of event $A$.

We assume, that the probability fulfills:

Axiom 4. $P(\Omega)=1$.

Axiom 5. $\forall A \in \mathcal{B}: P(A) \geq 0$.

Axiom 6. If $M=\left\{A_{n}\right\}$ is a finite or countable sequence of pairwise disjoint sets from $\mathcal{B}$, then

$$
P\left(\bigcup_{A_{n} \in M} A_{n}\right)=\sum_{A_{n} \in M} P\left(A_{n}\right)
$$

\section{Appendix - Proofs}

\subsection{The membership function $\boldsymbol{\mu}_{\boldsymbol{B}}(x)$ and the probability $Q(B)$ are well defined}

Lemma 3. For all $x \in X$ and for each set $\boldsymbol{B} \subseteq \boldsymbol{S}$, the sum $\boldsymbol{\mu}_{\boldsymbol{B}}(x)=\sum_{A_{i} \in \boldsymbol{B}} \mu_{A_{i}}(x)$ converges absolutely to some number from the interval $\langle 0,1\rangle$.

First, we prove the absolute convergence:

We can write $i<n$ where $n$ is a natural number and $i$ is an index of a fuzzy set $A_{i}$. (Assumption 1, Subsection 3.1.)

A sequence of partial sums $\sum_{A_{i} \in \boldsymbol{B} \wedge i<n} \mu_{A_{i}}(x)$ is non-decreasing because we add only non-negative functions. (Assumption 2, Subsection 3.1.)

The sequence of partial sums $\sum_{A_{i} \in \boldsymbol{B} \wedge i<n} \mu_{A_{i}}(x)$ is bounded above by number 1:

$$
\sum_{A_{i} \in \boldsymbol{B} \wedge i<n} \mu_{A_{i}}(x) \leq \sum_{A_{i} \in \boldsymbol{B}} \mu_{A_{i}}(x) \leq \sum_{A_{i} \in \boldsymbol{S}} \mu_{A_{i}}(x)=1,
$$

\footnotetext{
${ }^{4} \bar{A}$ is the designation for the set $\Omega-A$.
} 
where we used the Assumtion 3 in the last equation.

A finite limit of a non-decreasing, bounded from above sequence always exists, and thus the sum $\sum_{A_{i} \in \boldsymbol{B}} \mu_{A_{i}}(x)$ converges. (See e.g. [26], p. 55.)

The sum $\sum_{A_{i} \in \boldsymbol{B}} \mu_{A_{i}}(x)$ converges absolutely because we only add non-negative numbers.

Let us show that the sum $\sum_{A_{i} \in B} \mu_{A_{i}}(x)$ converges to some number in the interval $\langle 0,1\rangle$ :

The sum $\sum_{A_{i} \in \boldsymbol{B}} \mu_{A_{i}}(x)$ is equal to or greater than zero, because we add only non-negative numbers. (Assumption 2, Subsection 3.1.)

The sum $\sum_{A_{i} \in B} \mu_{A_{i}}(x)$ is equal to or smaller than one, because

$$
\sum_{A_{i} \in \boldsymbol{B}} \mu_{A_{i}}(x) \leq \sum_{A_{i} \in \boldsymbol{S}} \mu_{A_{i}}(x)=1
$$

where Assumtion 3 is used.

Lemma 4. The sum $\boldsymbol{\mu}_{\boldsymbol{B}}(x)=\sum_{A_{i} \in \boldsymbol{B}} \mu_{A_{i}}(x)$ does not depend on the order in which the addends are added together.

The sum $\sum_{A_{i} \in B} \mu_{A_{i}}(x)$ is a finite or countable sum according to Assumption 1, Subsection 3.1.

A finite sum does not depend on the order of addends.

If the sum is countable, then the expression $\sum_{A_{i} \in \boldsymbol{B}} \mu_{A_{i}}(x)$ converges absolutely according to Lemma 3. A sum of an absolutely convergent sequence does not depend on the order of addends too. (See e.g. [26], p. 99.)

Lemma 5. The function $\boldsymbol{\mu}_{B}(x)$ is measurable on the set $X$ with respect to the measure $P$. 3.1.)

The sum $\sum_{A_{i} \in \boldsymbol{B}} \mu_{A_{i}}(x)$ is a finite or countable sum (Assumption 1, Subsection

All functions $\mu_{A_{k}}$ are measurable on the set $X$ with respect to the measure $P$. (Assumption 5, Subsection 3.1.)

Finite series:

A sum of two measurable functions is a measurable function. (See e.g. [5], p. 24.) So, a finite sum of measurable functions is a measurable function. Therefore, if the sum $\boldsymbol{\mu}_{\boldsymbol{B}}(x)=\sum_{A_{i} \in \boldsymbol{B}} \mu_{A_{i}}(x)$ is a finite sum, then it is measurable.

Countable series:

If the sum $\boldsymbol{\mu}_{\boldsymbol{B}}(x)=\sum_{A_{i} \in B} \mu_{A_{i}}(x)$ is countable, then partial sums of it are measurable functions (according to the previous paragraph). A sequence of partial sums is non-decreasing. (Assumption 2, Subsection 3.1.) and a limit of a monotonous sequence of measurable functions is a measurable function. (See e.g. [5], p. 25.)

In both cases, the function $\boldsymbol{\mu}_{\boldsymbol{B}}(x)$ is measurable.

Lemma 6. The Lebesgue integral $Q(B)=\int_{X} \boldsymbol{\mu}_{\boldsymbol{B}}(x) d P$ always exists.

The function $\boldsymbol{\mu}_{\boldsymbol{B}}(x)$ is bounded (according to Lemma 3) and measurable on the set $X$ with respect to the measure $P$ (according to Lemma 5). 
$(X, \mathcal{A}, P)$ is a space with a probability measure. (Assumption 4, Subsection 3.1.) Therefore, it satisfies all Kolmogorov's axioms, especially Axiom 4. So $P(X)=1$. Therefore, the set $X$ is measurable with respect to the measure $P$ and its measure is finite.

A Lebesgue integral of bounded measurable function over a measurable set with a finite measure always exists. (See e.g. [5], pp. 29-31.)

\subsection{The basic floppy probability space meets all Kolmogorov's axioms}

Proposition 7. $\mathcal{P}(\boldsymbol{S})$ satisfies Axiom 1.

The power set $\mathcal{P}(\boldsymbol{S})$ contains all subsets of $\boldsymbol{S}$ and therefore specially $\varnothing$. So, Axiom 1 is fulfilled.

Proposition 8. $\mathcal{P}(\boldsymbol{S})$ meets Axiom 2.

A set of elements of $\boldsymbol{S}$ that do not belong to $\boldsymbol{B}$, is a subset of $\boldsymbol{S}$. The power set $\mathcal{P}(\boldsymbol{S})$ contains all subsets of $\boldsymbol{S}$ and therefore specifically $\overline{\boldsymbol{B}}$. So, Axiom 2 is fulfilled.

Proposition 9. $\mathcal{P}(\boldsymbol{S})$ fulfills Axiom 3.

An union of any system of subsets $\boldsymbol{S}$ is a subset of $\boldsymbol{S}$. The power set $\mathcal{P}(\boldsymbol{S})$ contains all subsets of $\boldsymbol{S}$ and therefore specifically $\bigcup_{i=1}^{\infty} \boldsymbol{B}_{\boldsymbol{i}}$ where $\boldsymbol{B}_{\boldsymbol{i}} \in \mathcal{P}(\boldsymbol{S})$. So, Axiom 3 is fulfilled.

Proposition 10. $(\boldsymbol{S}, \mathcal{P}(\boldsymbol{S}), Q)$ satisfies Axiom 4 .

Axiom 4 applies because:

$$
Q(\boldsymbol{S})=\int_{X} \boldsymbol{\mu}_{\boldsymbol{S}}(x) d P=\int_{X} \sum_{A_{i} \in \boldsymbol{S}} \mu_{A_{i}}(x) d P=\int_{X} 1 d P=P(X)=1
$$

where we used Assumtion 3 at first and then Axiom 4 for probability space $(X, \mathcal{A}, P)$ (according to Assumption 4, Subsection 3.1).

Proposition 11. $(\boldsymbol{S}, \mathcal{P}(\boldsymbol{S}), Q)$ satisfies Axiom 5.

A number $Q(\boldsymbol{B})$ exists for every set $\boldsymbol{B}$. (Lemma 6.) The number $Q(\boldsymbol{B})=$ $\int_{X} \boldsymbol{\mu}_{\boldsymbol{B}}(x) d P$ is non-negative because we integrate a non-negative function (according to Lemma 3.)

So, the space $(\boldsymbol{S}, \mathcal{P}(\boldsymbol{S}), Q)$ satisfies Axiom 5 .

Proposition 12. $(\boldsymbol{S}, \mathcal{P}(\boldsymbol{S}), Q)$ meats Axiom 6 .

Let $M=\left\{\boldsymbol{B}_{n}\right\}$ be a finite or countable sequence of pairwise disjoint sets from $\mathcal{P}(\boldsymbol{S})$. So, each fuzzy set $A_{k} \in \bigcup_{\boldsymbol{B}_{\boldsymbol{n}} \in M} \boldsymbol{B}_{\boldsymbol{n}}$ is an element of just one floppy set $\boldsymbol{B}_{\boldsymbol{n}}$.

Thus, we can write: 


$$
\begin{aligned}
Q\left(\bigcup_{\boldsymbol{B}_{n} \in M} \boldsymbol{B}_{\boldsymbol{n}}\right) & =\int_{X} \boldsymbol{\mu}_{\boldsymbol{B}_{\boldsymbol{n}} \in M} \boldsymbol{B}_{\boldsymbol{n}}(x) d P= \\
& =\int_{X} \sum_{A_{k} \in \cup \boldsymbol{B}_{\boldsymbol{n}}} \mu_{A_{k}}(x) d P= \\
& =\int_{X} \sum_{\boldsymbol{B}_{n} \in M}\left[\sum_{A_{k} \in \boldsymbol{B}_{n}} \mu_{A_{k}}(x)\right] d P= \\
& =\int_{X} \sum_{\boldsymbol{B}_{n} \in M} \boldsymbol{\mu}_{\boldsymbol{B}_{n}}(x) d P .
\end{aligned}
$$

We can exchange the sum and the integral because $\boldsymbol{\mu}_{\boldsymbol{B}_{n}}(x)$ are non-negative and measurable functions according to Lemmas 3 and 5. (See e.g. [16], p. 106.)

Therefore:

$$
Q\left(\bigcup_{\boldsymbol{B}_{n} \in M} \boldsymbol{B}_{\boldsymbol{n}}\right)=\sum_{\boldsymbol{B}_{n} \in M} \int_{X} \boldsymbol{\mu}_{\boldsymbol{B}_{n}}(x) d P=\sum_{\boldsymbol{B}_{n} \in M} Q\left(\boldsymbol{B}_{\boldsymbol{n}}\right) .
$$

So, we have proved that the space $(\boldsymbol{S}, \mathcal{P}(\boldsymbol{S}), Q)$ satisfies Axiom 6 .

\subsection{The floppy probability space meats all Kolmogorov's ax- ioms}

Proposition 13. $\mathcal{C}$ satisfies Axioms 1, 2 and 3.

$\mathcal{C}$ is a $\sigma$-algebra. (Definition 5 , Subsection 4.1.)

Proposition 14. $(\boldsymbol{S} \times X, \mathcal{C}, R)$ satisfies Axiom 4 .

$$
R(\boldsymbol{S} \times X)=\int_{X} \boldsymbol{\mu}_{\boldsymbol{S} \times \boldsymbol{X}}(x) d P=\int_{X} \sum_{A_{i} \in \boldsymbol{S}:\left[A_{i}, x\right] \in \boldsymbol{S} \times X} \mu_{A_{i}}(x) d P=\int_{X} 1 d P=1 .
$$

Assumtion 3 and then Axiom 4 for probability space $(X, \mathcal{A}, P)$ (according to Assumption 4, Subsection 3.1) were used.

Proposition 15. $(\boldsymbol{S} \times X, \mathcal{C}, R)$ satisfies Axiom 5 .

We can write:

$$
R(\boldsymbol{C})=\int_{X} \boldsymbol{\mu}_{\boldsymbol{C}}(x) d P=\int_{X} \sum_{A_{i} \in \boldsymbol{S}:\left[A_{i}, x\right] \in \boldsymbol{C}} \mu_{A_{i}}(x) d P .
$$

$\mu_{A_{i}}(x)$ is measurable on the set $\left\{x \in X:\left[A_{i}, x\right] \in C\right\}$ (Assumption 5, Subsection 3.1) and non-negative (Assumption 2, Subsection 3.1). Thus, the sum $\sum_{A_{i} \in \boldsymbol{S}:\left[A_{i}, x\right] \in \boldsymbol{C}} \mu_{A_{i}}(x)$ is measurable and non-negative. So, the integral $R(\boldsymbol{C})=$ $\int_{X} \sum_{A_{i} \in \boldsymbol{S}:\left[A_{i}, x\right] \in \boldsymbol{C}} \mu_{A_{i}}(x) d P$ exists and is non-negative. 
Proposition 16. $(\boldsymbol{S} \times X, \mathcal{C}, R)$ satisfies Axiom 6 . $\mathcal{C}$.

Let $M=\left\{\boldsymbol{C}_{n}\right\}$ be a finite or countable sequence of pairwise disjoint sets from

Thus, each point $\left[A_{i}, x\right] \in \bigcup_{C_{n} \in M} \boldsymbol{C}_{\boldsymbol{n}}$ is an element of just one floppy set $\boldsymbol{C}_{\boldsymbol{n}}$.

Therefore, we can write:

$$
\begin{aligned}
& R\left(\bigcup_{C_{n} \in M} \boldsymbol{C}_{\boldsymbol{n}}\right)=\int_{X} \boldsymbol{\mu} \cup_{C_{n} \in M} \boldsymbol{C}_{n}(x) d P= \\
& =\int_{X} \sum_{A_{i} \in S:\left[A_{i}, x\right] \in} \bigcup_{C_{n} \in M} \mu_{A_{i}}(x) d P= \\
& =\int_{X} \sum_{\boldsymbol{C}_{\boldsymbol{n}} \in M}\left[\sum_{A_{i} \in \boldsymbol{S}:\left[A_{i}, x\right] \in \boldsymbol{C}_{\boldsymbol{n}}} \mu_{A_{i}}(x)\right] d P= \\
& =\int_{X} \sum_{\boldsymbol{C}_{n} \in M} \boldsymbol{\mu}_{C_{n}}(x) d P \text {. }
\end{aligned}
$$

We can exchange the sum and the integral because $\boldsymbol{\mu}_{\boldsymbol{C}_{n}}(x)$ are non-negative and measurable functions.

Therefore:

$$
R\left(\bigcup_{\boldsymbol{C}_{\boldsymbol{n}} \in M} \boldsymbol{C}_{\boldsymbol{n}}\right)=\sum_{\boldsymbol{C}_{\boldsymbol{n}} \in M} \int_{X} \boldsymbol{\mu}_{\boldsymbol{C}_{\boldsymbol{n}}}(x) d P=\sum_{\boldsymbol{C}_{\boldsymbol{n}} \in M} R\left(\boldsymbol{C}_{\boldsymbol{n}}\right) .
$$

So, we have proved that the space $(\boldsymbol{S} \times X, \mathcal{C}, R)$ satisfies Axiom 6 .

\section{References}

[1] BARDUK R. The new upper bound on the probability of error in a binary tree classifier with fuzzy information. Neural Network World. 2010, 20, pp. 951-961. ISSN 1210-0552.

[2] BELLMAN R, KALABA R, ZADEH L. Abstraction and pattern classification. Journal of Mathematical Analysis and Applications. 1966, 13(1), pp. 1-7, doi: 10.1016/0022-247X(66) 90071-0. ISSN 0022247x.

[3] BELLMAN R.E., ZADEH L.A. Decision-Making in a Fuzzy Environment. Manage- ment Science. 1970, 17(4), pp. B-141-B-164, doi: 10.1287/mnsc.17.4.B141. ISSN 0025-1909.

[4] BOUChon-Meunier B., DUBOiS D., GODO L., PRADE H. Fuzzy Sets and Possibility Theory in Approximate and Plausible Reasoning. In: Fuzzy sets in approximate reasoning and information systems. Boston, MA: Springer, 1999, pp. 15-190. ISBN 9781461552437.

[5] BURKILL J.C. The Lebesgue integral. London: Cambridge University Press, 1963.

[6] DUBOIS D., PRADE H. Fuzzy sets and probability: misunderstandings, bridges and gaps. In: [Proceedings 1993] Second IEEE International Conference on Fuzzy Systems, San Francisco, CA: IEEE, 1993, pp. 1059-1068, doi: 10.1109/FUZZY.1993.327367. ISBN 0-7803-06147 .

[7] DUBOIS D., PRADE H. Possibility Theory. Wiley Encyclopedia of Electrical and Electronics Engineering. 2001-08-21, doi: 10.1002/047134608X.W3502. 


\section{Provinský P.: Floppy logic - a younger sister of fuzzy logic}

[8] DUBOIS D., PRADE H.M. Fundamentals of fuzzy sets. Boston: Kluwer Academic, 2000. ISBN 07-923-7732-X.

[9] DUBOIS D., PRADE H.M. Fuzzy sets and systems: theory and applications. New York: Academic Press, 1980. ISBN 01-222-2750-6.

[10] Fuzzy logic. Wikipedia: the free encyclopedia, San Francisco (CA): Wikimedia Foundation, 2015 [wieved 2015-06-09]. Available also from: http://en.wikipedia.org/wiki/Fuzzy_ logic.

[11] GAINES B.R. Fuzzy and probability uncertainty logics. Information and Con- trol. 1978, 38(2), pp. 154-169, doi: 10.1016/S0019-9958(78)90165-1. ISSN 00199958.

[12] GILES R. Lukasiewicz logic and fuzzy set theory. International Journal of Man- Machine Studies. 1976, 8(3), pp. 313-327, doi: 10.1016/S0020-7373(76)80003-X. ISSN 00207373.

[13] GODO L., ESTEVA F., HÁJEK P. Reasoning about probability using fuzzy logic. Neural Network World. 2000, 10(5), pp. 811-824. ISSN 1210-0552.

[14] HERSH H.M., CARAMAZZA A. A fuzzy set approach to modifiers and vagueness in natural language. Journal of Experimental Psychology: General. 1976, 105(3), pp. 254-276, doi: 10. 1037/0096-3445.105.3.254. ISSN 0096-3445.

[15] KOLMOGOROV A.N. Grundbegriffe der Wahrscheinlichkeitsrechnung (Foundations of the Theory of Probability). Berlin: J. Springer, 1933.

[16] KOPÁČEK J. Matematická analýza nejen pro fyziky (III) (Mathematical Analysis Not Only for Physicists (III). 3., upr. vyd. Praha: Matfyzpress, 2007. ISBN 978-80-7378-020-3.

[17] KOSKO B. Fuzziness vs. Probability. International Journal of General Systems. 1990, 17(23), pp. 211-240, doi: 10.1080/03081079008935108. ISSN 0308-1079.

[18] MAISTROV L.E. Probability theory: a historical sketch. New York: Academic Press, 1974 ISBN 01-246-5750-8.

[19] MENGER K. Statistical Metrics. Proceedings of the National Academy of Sciences of the United States of America. 1942, 28(12), pp. 535-537, doi: 10.1073/pnas.28.12.535.

[20] MONTES I., HERNÁNDEZ J., MARTINETTI D., MONTES S. Characterization of continuous t-norms compatible with Zadeh's probability of fuzzy events. Fuzzy Sets and Systems. 2013, 228(October, 2013), pp. 29-43, doi: 10.1016/j.fss.2012.11.020. ISSN 01650114.

[21] OKUDA T., TANAKA H., ASAI K. A formulation of fuzzy decision problems with fuzzy information using probability measures of fuzzy events. Information and Control. 1978, 38(2), pp. 135-147, doi: 10.1016/S0019-9958(78)90151-1. ISSN 00199958.

[22] RESCHER N. Many-valued logic. New York: McGraw-Hill, 1969. ISBN 0070518939.

[23] RÉNYI A. On a new axiomatic theory of probability. Acta Mathematica Academiae Scientiarum Hungaricae. 1955, 6(3-4), pp. 285-335, doi: 10.1007/BF02024393. ISSN 0001-5954.

[24] STIGLER S.M. The history of statistics: the measurement of uncertainty before 1900. Cambridge, Mass.: Belknap Press of Harvard University Press, 1986. ISBN 06-744-0340-1.

[25] ŠTĚPÁN J. Teorie pravděpodobnosti: Matematické základy (Probability Theory: Mathematical Foundations. Praha: Academia, 1987.

[26] VESELÝ J. Základy matematické analýzy: Prvnídí (Fundamentals of Mathematical Analysis: First Part). Praha: Matfyzpress, 2004. ISBN 978-80-7378-063-02.

[27] ZADEH L.A. Fuzzy probabilities. Information Processing. 1984, 20(3), pp. 363-372, doi: 10. 1016/0306-4573(84)90067-0. ISSN 03064573.

[28] ZADEH L.A. Fuzzy sets. Information and Control. 1965, 8(3), pp. 338-353, doi: 10.1016/ S0019-9958(65)90241-X. ISSN 00199958.

[29] ZADEH L.A. Fuzzy sets as a basis for a theory of possibility. Fuzzy Sets and Systems. 1978, 1(1), pp. 3-28, doi: 10.1016/0165-0114(78)90029-5. ISSN 01650114.

[30] ZADEH L.A. Probability Measures of Fuzzy Events. Journal of Mathematical Anal- ysis and Applications. 1968, 23(2), pp. 421-427, doi: 10.1016/0022-247X(68)90078-4. ISSN $0022247 \mathrm{x}$ 